\title{
Suppressing bladder artifacts in bone SPECT of the pelvis
}

\author{
Gilles Allenbach $\cdot$ John O. Prior $\cdot$ Nicolas Theumann \\ Nessria El-Hasnawy $\cdot$ Jerome Malterre \\ Angelika B. Delaloye $\cdot$ Ehab M. Kamel
}

Received: 31 July 2006 / Accepted: 16 April 2007

(C) The Japanese Society of Nuclear Medicine 2007

\begin{abstract}
Objective Bladder-filling reconstruction artifacts have a detrimental effect on the image quality of pelvic bone single photon emission computed tomography (SPECT). Using a simple protocol consisting of forced diuresis coupled with intravenous (IV) hydration, this study was undertaken to obtain an artifact-free pelvic SPECT after discarding the residual urinary activity.

Methods Thirty patients were enrolled. In group I, pelvic SPECT was performed directly after normal void, whereas in group II, SPECT was preceded by IV injection of $0.5 \mathrm{mg} / \mathrm{kg}$ furosemide (maximum $40 \mathrm{mg}$ ) coupled with IV infusion of $500 \mathrm{cc}$ of physiologic saline. Bladderfilling reconstruction artifacts were analyzed in group I patients, who had their images reconstructed using both filtered backprojection and iterative algorithms, both qualitatively and quantitatively by means of regions of interest (ROIs) drawn around the artifact-bearing bone areas as well as the corresponding contralateral sites. For group II patients, besides visual analysis, ROIs were placed over the sites corresponding to those of the group I patients. In every patient, total counts of each ROI were normalized to a reference ROI placed over the sacrum, and a ratio was created.

Results Using filtered backprojection, two forms of artifacts were identified in group I patients: first, a streak
\end{abstract}

G. Allenbach · J. O. Prior · N. El-Hasnawy $\cdot$ J. Malterre ·

A. B. Delaloye $\cdot$ E. M. Kamel $(\triangle)$

Division of Nuclear Medicine, Centre Hospitalier Universitaire

Vaudois (CHUV), CH-1011 Lausanne, Switzerland

e-mail: Mohamed-Ehab.Kamel@chuv.ch

N. Theumann

Department of Diagnostic and Interventional Radiology, Centre Hospitalier Universitaire Vaudois (CHUV), Lausanne,

Switzerland pattern that extended to the sacro-iliac joint in nine $(60 \%)$ patients, the hip joint in five $(33 \%)$, the superior pubic rami in four $(27 \%)$, the sacrum in three $(20 \%)$, and the ischium in one $(6 \%)$; second, a count loss subtype which extended to the hip joints in nine $(60 \%)$ patients. Corresponding values after iterative reconstruction were two $(13 \%)$ for the sacro-iliac joint, three $(20 \%)$ for the hip joint, one $(6 \%)$ for the superior pubic ramus, and one $(6 \%)$ for the sacrum. In five $(33 \%)$ patients, residual count loss artifacts were still identifiable after iterative reconstruction. However in group II, no such effects were observed because the bladder activity reached near background level in $14(93 \%)$ of 15 patients after three successive voids with a 3.5 -fold decrease in the mean value of total bladder count in comparison with group I patients. A statistically significant difference was found between artifact- and non-artifact-harboring ROIs in group I whichever the method used for reconstruction, whereas the values of right and left hemi-pelvis ROIs/ sacrum in group II were almost identical.

Conclusions Forced diuresis coupled with parenteral hydration facilitates the acquisition of an artifact-free pelvic SPECT. Especially for clinical questions that focus on femoral heads and pubic bones, applying the aforementioned protocol may improve the diagnostic accuracy of pelvic bone SPECT.

Keywords Bladder artifacts $\cdot$ Forced diuresis $\cdot$ Pelvic bone SPECT

\section{Introduction}

Bladder-filling reconstruction artifacts represent an obvious limitation of pelvic single photon emission com- 
puted tomography (SPECT). These artifacts may negate the diagnostic accuracy of bone SPECT in identifying many pathologic processes that originate from the pelvic girdle. Besides the well-recognized streak artifacts that may mimic or mask pathologic hot spots, count loss artifacts, which appear as photopenic zones in structures within the same transverse plane of a filled bladder, can resemble or obscure the diagnosis of femoral head avascular necrosis [1]. Furthermore, both pubic bones and the superior pubic rami can be simply rendered uninterpretable by virtue of a full bladder. Encouraging patients to void before undergoing pelvic SPECT does not guarantee an artifact-free examination because the bladder refills throughout the acquisition period [1, 2].

Continuous efforts, with variable rates of success, have been devoted to overcome these limitations inherent to pelvic bone SPECT [3-6]. However, to date, these attempts have invariably proposed software-derived techniques that besides their relative complexity and limited availability, were not always satisfactory. On the basis of this background, an efficient and practical solution that radically tackles artifact creation in pelvic SPECT would be of great benefit.

Using a simple protocol consisting of forced diuresis coupled with intravenous (IV) hydration, this study was undertaken to obtain an artifact-free pelvic SPECT after discarding the residual urinary activity. For this purpose, we have prospectively examined a cohort of patients who were referred to our institution with various clinical problems primarily related to the bony pelvis.

\section{Materials and methods}

Thirty patients divided equally into two groups (16 men, 14 women; age range $18-83$ years; mean age 59 years) were enrolled in the present study. Because our main objective was to validate a new protocol to overcome the problem of bladder-filling reconstruction artifacts, we retrospectively selected 15 patients (group I) in whom pelvic SPECT was done without any pretest patient preparation to serve as an artifact model. In this patient cohort, a full bladder in the tomography raw data was considered to be the primary selection criteria that can resemble the worst scenario of artifact creation after image reconstruction.

On the other hand, another 15 consecutive patients (group II) presenting with pain or discomfort in the pelvic girdle were subjected to IV injection of $0.5 \mathrm{mg} / \mathrm{kg}$ furosemide (maximum $40 \mathrm{mg}$ ) coupled with parenteral infusion of $500 \mathrm{cc}$ of physiologic saline over $25-30 \mathrm{~min}$ before their scheduled pelvic SPECT [7]. During this phase, patients were also encouraged to drink two cups
$(400 \mathrm{cc})$ of water besides their continuous parenteral saline infusion. Furthermore, arterial blood pressure was monitored in each patient every $10 \mathrm{~min}$, and the last value was reported just before starting the SPECT acquisition. Patients with known cardiac decompensation, renal insufficiency, or obstructive uropathy were excluded from the present study.

Each patient was injected with $600 \mathrm{MBq}$ of ${ }^{99 \mathrm{~m}}$ technetium-dicarboxypropane diphosphonate ( $\left.{ }^{99 \mathrm{~m}} \mathrm{Tc}-\mathrm{DPD}\right)$ intravenously, and then tomographic data acquisition was started $4 \mathrm{~h}$ after injection using a three-headed Triad XLT gamma camera (Trionix, Twinsburg, OH, USA) equipped with low-energy all-purpose collimators. In our department, patients are always asked to empty their bladder before undergoing the acquisition. Data were collected using a circular orbit with 60 angles over $360^{\circ}$ and an acquisition period of $30 \mathrm{~s}$ per angle. The patients were scanned in the supine position, and the starting position corresponded to a posterior view. A $128 \times 128$ matrix was used for data storage, and reconstruction was performed using filtered backprojection with a Butterworth filter and cutoff of 0.5 cycle/pixel. For group I patients, iterative reconstruction (ordered subsets expectation maximization with two iterations and 10 subsets) was also performed to evaluate the additional value of this algorithm in suppressing the bladder-filling reconstruction artifacts. No scatter or attenuation correction was performed.

After image reconstruction, streak artifacts were analyzed in group I subjects, who had their images reconstructed using both filtered backprojection and iterative algorithms, both qualitatively (for location and extent) by two nuclear medicine physicians in consensus and quantitatively by means of regions of interest (ROIs) placed over the artifact-bearing bone regions as well as around the corresponding contralateral sites, provided they were artifact free (Fig. 1a, b). Count loss artifacts, however, were analyzed only qualitatively because such artifacts have a bilateral and symmetric pattern in most instances. For each patient in group II, besides visual analysis of any potential streak or count loss artifacts, ROIs were drawn over sites corresponding to those of one different patient of group I (Fig. 1c). This approach was chosen to standardize the process of ROIs placement between the two different patient groups. Areas with pathologic hot spots were not considered for analysis.

In every patient, total counts of each ROI were normalized to that of a reference ROI drawn over the lower part of the first and upper part of the second-sacral vertebra, and a ratio was created. This reference region was chosen because it is theoretically remote from any bladder-filling reconstruction artifacts and is hardly 
Fig. 1 Transaxial single photon emission computed tomography (SPECT) of the pelvis displays characteristic streak and count loss artifacts caused by a full bladder after filtered backprojection (a), and substantial amelioration after iterative reconstruction (b). Transaxial SPECT of the pelvis in another patient who was subjected to furosemide challenge shows excellent image quality (c).

Comparative plots of artifactbearing regions of interest (ROIs)/sacrum versus that of artifact-free ROIs/sacrum in group I using filtered backprojection (d), iterative reconstruction (e), and corresponding ROIs/sacrumderived values in group II (f)
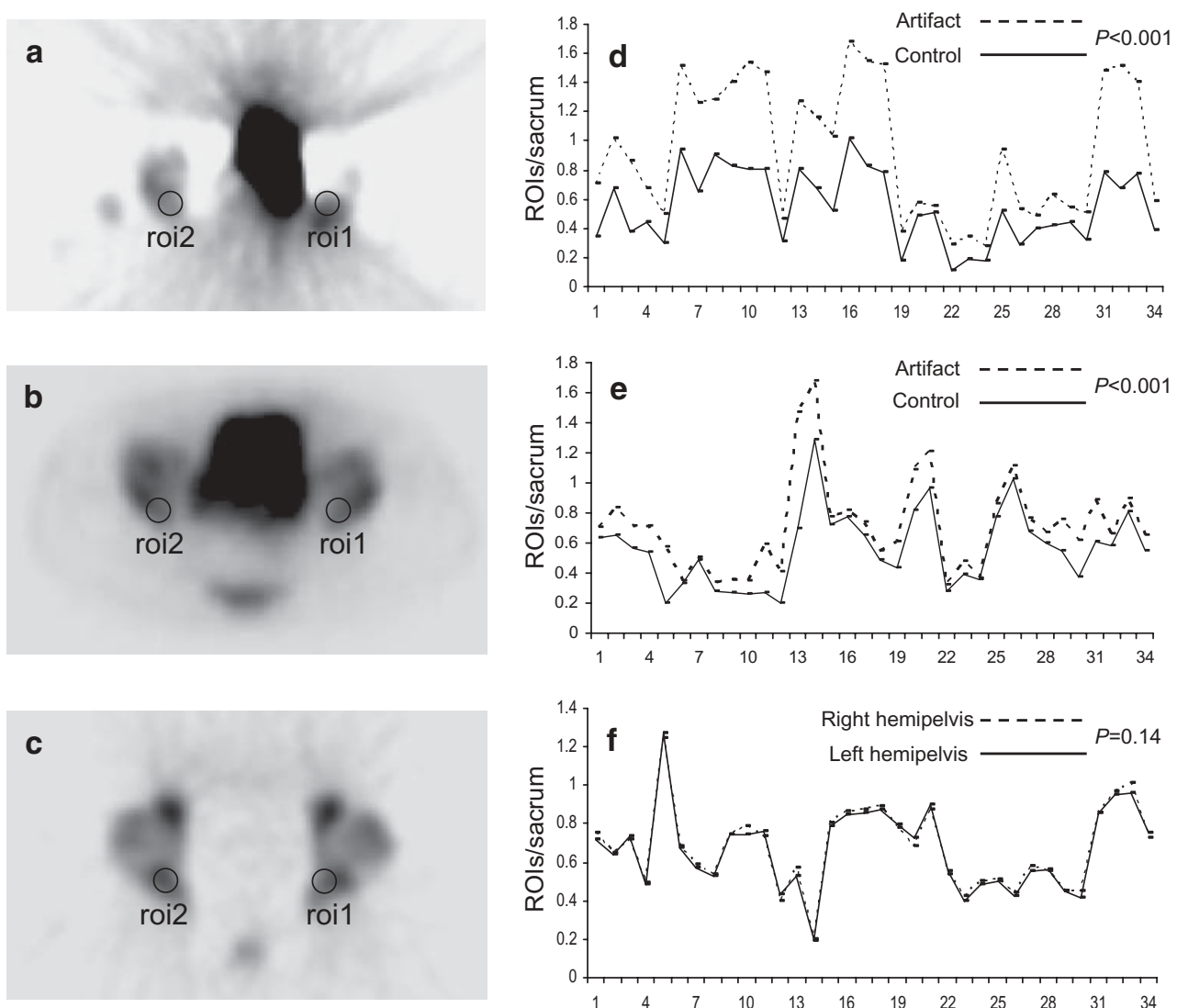

affected by degenerative joint diseases owing to its relative immobility. In both patient groups, a paired $t$ test was performed to compare the ratios of each couple of ROIs with the sacrum. A $P$-value of less than 0.05 was considered to be significant.

\section{Results}

In the studied patient population, forced diuresis coupled with parenteral hydration was proved to be safe, easy to apply, and tolerable. No patient developed any untoward effect in the form of hypotension, allergic reaction, or fluid overload. In group I $(n=15)$, bladder-filling reconstruction streak artifacts, which had a random and unpredictable nature, extended to the lower part of the sacro-iliac joint in nine $(60 \%)$ patients, hip joint in five $(33 \%)$, superior pubic ramus in four $(27 \%)$, caudal part of the sacrum in three $(20 \%)$, and ischium in one $(6 \%)$. Corresponding values after iterative reconstruction were two $(13 \%)$ for the sacro-iliac joint, three $(20 \%)$ for the hip joint, one $(6 \%)$ for the superior pubic ramus, and one $(6 \%)$ for the caudal part of the sacrum. Additionally, count loss artifacts, which invariably extended to the hip joints on either side, were observed in nine $(60 \%)$ patients after filtered backprojection and in five (33\%) after iterative reconstruction. This form of artifact provoked bilateral photon deficient zones that precluded confident interpretation of the anatomic structures located along its path (Fig. 1a). In eight (53\%) patients, both pubic bones and the superior pubic rami were uninterpretable because they were masked by activity from the distended bladder despite the applied algorithms for image reconstruction.

In group II $(n=15)$, no such effects were observed because the bladder activity was reduced to a near background level after three successive voids in $14(93 \%)$ of 15 patients (Fig. 1c). Interestingly, a 3.5-fold reduction in the mean value of the total bladder count in group II patients has been observed in comparison with group I (mean value of total bladder count 128600 vs. 426066 , $P<0.001)$. One patient who voided only twice before SPECT acquisition revealed some residual urinary activity that did not interfere with the diagnosis of a fissure fracture of the femoral neck or masked his pubic bones (Fig 2). In that patient, no significant artifacts could be recognized. In the remaining 14 patients, 2 were diagnosed with fractures of the femur neck with $(n=1)$ or without $(n=1)$ avascular necrosis of the femoral head. Three patients were identified with fractures of the 

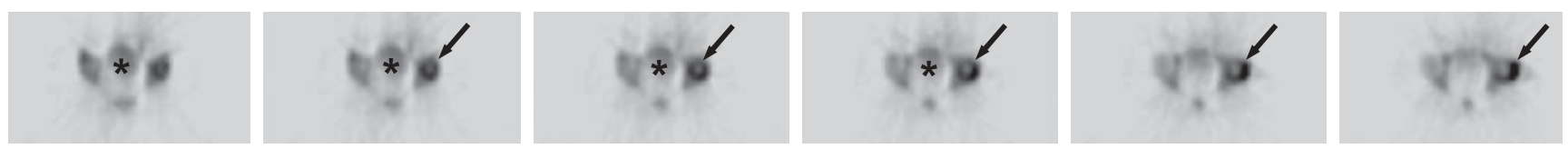

Fig. 2 Transaxial SPECT of the pelvis in a 48-year-old male patient shows residual urinary activity (asterisks) after his bladder had been emptied twice. This residual bladder activity, however,

sacrum $(n=2)$ and the superior pubic ramus $(n=1)$. Two patients displayed degenerative changes in the lumbosacral junction. In seven patients, no significant osseous pathology could be identified in the pelvic girdle.

For each patient group, a total of 34 couples of ROIs were available. Using filtered backprojection, statistical analysis revealed a significant difference between artifact- and non-artifact-harboring ROIs in group I. The mean ratios of artifact-bearing ROIs/sacrum versus that of artifact-free ROIs/sacrum were $0.92 \pm 0.46$ and $0.55 \pm 0.25(P<0.001)$, respectively (Fig. 1d). When iterative reconstruction was considered, statistical analysis revealed a significant difference between artifact- and non-artifact-harboring ROIs $(P<0.001)$ with mean ratios of artifact-bearing ROIs/sacrum versus that of artifact-free ROIs/sacrum of $0.70 \pm 0.31$ and $0.56 \pm 0.25$, respectively (Fig. 1e). On the other hand, the values of right and left hemi-pelvis ROIs/sacrum in group II were almost identical, showing mean values of $0.64 \pm 0.21$ and $0.63 \pm 0.20(P=0.14)$, respectively (Fig. 1f).

Interestingly, $14(93 \%)$ of 15 patients of the furosemide challenge group tolerated $30 \mathrm{~min}$ of SPECT imaging without an urgent need to void. Noteworthy, however, is that a young 21-year-old male patient was allowed to empty his bladder, in a urine container, $10 \mathrm{~min}$ before study termination. In that patient, no motion artifacts were identified on reviewing his final SPECT images.

\section{Discussion}

The problem of bladder-filling reconstruction artifacts, which may render approximately $20 \%$ of scans uninterpretable, has been addressed in many reports [3-6]. However, to date, there is no best solution that guarantees artifact-free pelvic SPECT. For instance, digital filtering of the bladder was attempted by Gillen et al. [3]. In this approach, the counts in the area occupied by the filled bladder were replaced by data that represent activity in the surrounding extra-vesical regions [3]. Although a marked improvement in image quality in the range of $78 \%-85 \%$ was reported, postprocessing image deterioration was observed in $1(8 \%)$ of 13 patients. In another more successful trial, Bunker et al. [4] reported on an image-reprocessing algorithm that reduced the technical neither cause considerable reconstruction artifacts nor interfere with the diagnosis of a fissure fracture in the left femoral neck (arrows)

inadequacy from $19 \%$ to $2 \%$ for the SPECT evaluation of 100 hips with suspected osteonecrosis or occult fracture. In this investigation, the highest pixel value associated with the osseous or periosseous areas of clinical interest was first defined in the raw data. In a second step, any pixel value "essentially in the bladder region" that contains counts greater than the highest value of interest was truncated using a special equation. However, it was further emphasized that the pixel-truncation technique leads to some distortion of the hips and can interfere with the accuracy of quantitative measurements because it underestimates the hip activities by $20 \%-30 \%$ [5].

Recently, Wells et al. [6] investigated dynamic SPECT (dSPECT) in 20 patients who presented with or without pain/discomfort in the hips. As the name implies, dSPECT is capable of estimating the kinetic parameters from studies performed using a single rotation acquisition of a standard SPECT camera. This technique implements the temporal information already present in the SPECT projection data in the reconstruction process. In other words, a series of images is reconstructed where each one corresponds to the activity distribution at the time when the projection is acquired. Consequently, the inconsistencies in the projection data, as the bladder activity changes throughout the acquisition time, and its sequels of artifact creation are both averted. Aside from the fact that dSPECT was proved to be very effective at removing bladder artifacts, however, this algorithm, that mandates the incorporation of either rotating source or computed tomography (CT)-based attenuation correction $[8,9]$, was shown to increase the acquisition time by $25 \%$ and did not allow confident interpretation of the symphysis pubis or the medial portion of both pubic rami [6]. Furthermore, unless a triple- or dual-head (with heads at $90^{\circ}$ ) camera is used, substantial streak artifacts could still be produced had dSPECT been obtained from single- or dual-head (with heads at $180^{\circ}$ ) systems $[8,10]$.

In the present study, we introduced a simple, efficient, and well-tolerated method to radically discard the bladder-filling reconstruction artifacts from bone SPECT of the pelvis. In $14(93 \%)$ of 15 patients who were subjected to forced diuresis, we observed that a dosage of $0.5 \mathrm{mg} / \mathrm{kg}$ furosemide (maximum $40 \mathrm{mg}$ ) coupled with 


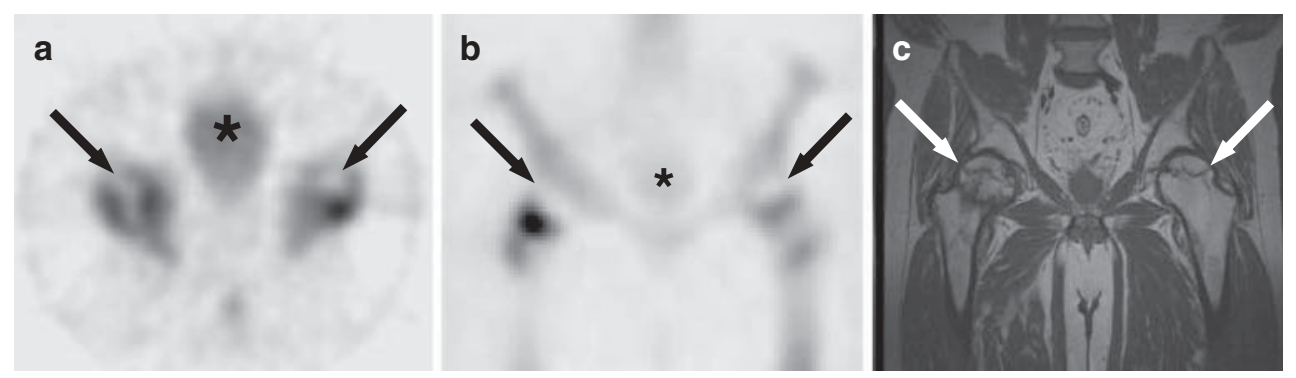

Fig. 3 Transaxial (a), and coronal (b) SPECT of the pelvis in a 61-year-old male patient, who presented with bilateral hip pain, reveal frank photopenia in the femoral head on either side (arrows) consistent with avascular necrosis that was confirmed by coronal
T1-weighted magnetic resonance imaging (c). The residual bladder activity (asterisks) after furosemide challenge is comparable with that of the background parenteral infusion of $500 \mathrm{cc}$ of physiologic saline over 25-30 min was quite successful in eliminating residual radioactivity from the urinary bladder. At least three successive voids were considered mandatory to reduce the urinary activity to a near background level without the need for a catheter to evacuate/irrigate the bladder (Fig. 1c). This was directly translated to an absolute suppression of both the reconstruction streak and count loss artifacts from the final SPECT images along with a potential reduction in the radiation exposure of pelvic organs in comparison with the other patient group $(n=15)$ that was not subjected to furosemide challenge.

Furthermore, statistical analysis of ROIs from both patient groups confirmed the results obtained from the visual interpretation where a statistically significant difference was found between artifact- and non-artifactharboring ROIs in group I regardless of the method used for reconstruction (Fig. 1d, e), whereas the values of right and left hemi-pelvis ROIs/sacrum in group II were almost identical (Fig. 1f). This may imply that the application of our protocol can guarantee an accurate quantitative analysis of SPECT studies that concern the bony pelvis.

Besides the unambiguous amelioration in the overall performance of postdiuretic pelvic bone SPECT especially in the detection of femoral head avascular necrosis (Fig. 3), these encouraging results may permit us to expect a similar rate of success at the level of F-18 fluoro-deoxyD-glucose positron emission tomography $\left({ }^{18} \mathrm{~F}\right.$-FDG PET $)$ and ${ }^{18} \mathrm{~F}$-choline PET where an obvious contrast handicap in the lower urinary tract is still challenging even in the face of the marked improvement provided by PET/CT $[11,12]$. In a recent publication, our group showed very promising results in this regard [7]. Likewise, with the increasing involvement of ${ }^{18} \mathrm{~F}$-fluoride PET $\left({ }^{18} \mathrm{~F}\right.$-PET) in the assessment of variable osseous pathologies [13, 14], applying the aforementioned protocol may also allow artifact-free ${ }^{18} \mathrm{~F}$-PET of the pelvis. This can be achieved without the need to insert a bladder catheter, which besides its impracticability and potential complications, tends to increase the radiation exposure to the SPECT/ PET personnel $[15,16]$.

When iterative reconstruction was applied [17], a dramatic reduction in artifact creation has been observed on qualitative analysis (Fig. 1b). Noteworthy, however, is that both pubic bones and the superior pubic rami were masked by the residual bladder activity in $8(53 \%)$ patients of group I regardless of the method used for reconstruction. This negative effect could be totally averted using our protocol (Fig. 4). Accordingly, the diagnosis of many traumatic, inflammatory and neoplastic disorders that originate from these osseous structures can be confidently made without the need to perform any delayed imaging that can be cumbersome especially for patients who are referred from remote areas.

\section{Conclusions}

In patients with adequate renal functions and preserved physiologic voiding, forced diuresis coupled with parenteral hydration facilitates the acquisition of artifacts-free pelvic SPECT. This holds true for both the streak and count loss subtypes. Three successive voids must be performed before bringing the residual urinary activity to a near background level without the need for a catheter to evacuate/irrigate the bladder. The application of iterative reconstruction has the potential to dramatically reduce, but cannot totally suppress, the bladder-filling reconstruction artifacts. Furthermore, it does not guarantee the confident interpretation of both pubic bones and the superior pubic rami. Taken together, our practical approach may have a major positive impact on the overall diagnostic accuracy of bone SPECT studies of the pelvis. 

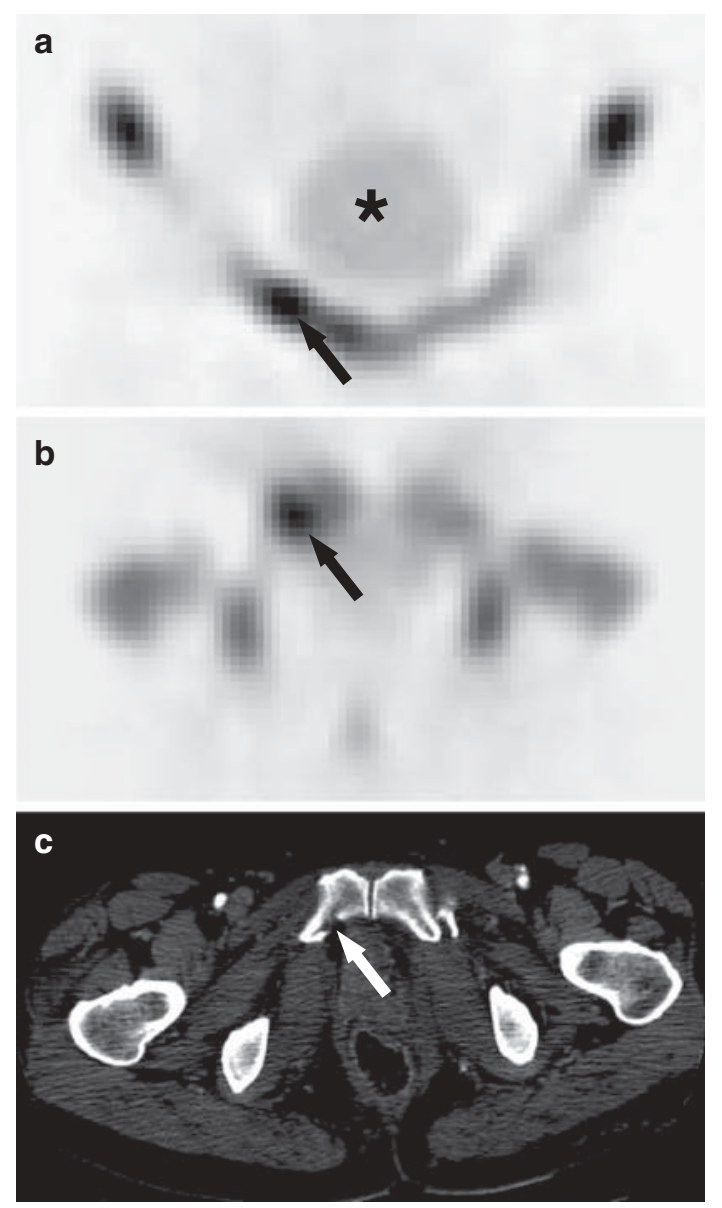

Fig. 4 Coronal (a), and transaxial (b) SPECT of the pelvis in a 57-year-old female patient after a traffic accident display a hot spot in the right superior pubic ramus (arrows) suggesting a fracture that was confirmed by CT (c). The residual bladder activity (asterisk) after furosemide challenge is very close to that of the background

Acknowledgments Dr El-Hasnawy is supported by the International Atomic Energy Agency (IAEA), scholarship C6/MOR/ 03017P. This article was presented in part at the 52nd Annual Meeting of the Society of Nuclear Medicine, Toronto, ON, Canada, June 19-23, 2005. The authors gratefully acknowledge the advice of Ariane Boubaker, MD, and the technical assistance of Chantal Séverin, Jacqueline Rivar, Murielle Croisier, and Eric Chevallier.

\section{References}

1. Gillen GJ, Gilmore B, Elliott AT. An investigation of the magnitude and causes of count loss artifacts in SPECT imaging. J Nucl Med 1991;32:1771-6.
2. Collier BD, Carrera GF, Johnson RP, Isitman T, Hellman RS, Knobel J, et al. Detection of femoral head avascular necrosis in adults by SPECT. J Nucl Med 1985;26:979-87.

3. Gillen GJ, McKillop JH, Hilditch TE, Davidson JK, Elliott AT. Digital filtering of the bladder in SPECT bone studies of the pelvis. J Nucl Med 1988;29:1587-95.

4. Bunker SR, Handmaker H, Torre DM, Schmidt WP. Pixel overflow artifacts in SPECT evaluation of the skeleton. Radiology 1990;174:229-32.

5. O'Connor MK, Kelly BJ. Evaluation of techniques for the elimination of "hot" bladder artifacts in SPECT of the pelvis. J Nucl Med 1990;31:1872-5.

6. Wells RG, Farncombe T, Chang E, Nicholson RL. Reducing bladder artifacts in clinical pelvic SPECT images. J Nucl Med 2004:45:1309-14.

7. Kamel EM, Jichlinski P, Prior JO, Meuwly J-Y, Delaloye J-F, Vaucher L, et al. Forced diuresis improves the diagnostic accuracy of ${ }^{18} \mathrm{~F}$-FDG PET in abdominopelvic malignancies. J Nucl Med 2006;47:1803-7.

8. Celler A, Farncombe T, Bever C, Noll D, Maeght J, Harrop $\mathrm{R}$, et al. Performance of the dynamic single photon emission computed tomography (dSPECT) method for decreasing or increasing activity changes. Phys Med Biol 2000;45:3525-43.

9. Utsunomiya D, Tomiguchi S, Shiraishi S, Yamada K, Honda $\mathrm{T}$, Kawanaka K, et al. Initial experience with X-ray CT based attenuation correction in myocardial perfusion SPECT/CT system. Ann Nucl Med 2005;19:485-9.

10. Farncombe T, Celler A, Noll D, Maeght J, Harrop R. Dynamic SPECT imaging using a single camera rotation (dSPECT). IEEE Trans Nucl Sci 1999;46:1055-61.

11. Schoder H, Larson SM. Positron emission tomography for prostate, bladder, and renal cancer. Semin Nucl Med 2004;34:274-92.

12. Schmid DT, John H, Zweifel R, Cservenyak T, Westera G, Goerres GW, et al. Fluorocholine PET/CT in patients with prostate cancer: initial experience. Radiology 2005;235: 623-8.

13. Hetzel M, Arslandemir C, Konig HH, Buck AK, Nussle K, Glatting G, et al. F-18 NaF PET for detection of bone metastases in lung cancer: accuracy, cost-effectiveness, and impact on patient management. J Bone Miner Res 2003;18:2206-14.

14. Brenner W, Vernon C, Muzi M, Mankoff DA, Link JM, Conrad EU, et al. Comparison of different quantitative approaches to 18F-fluoride PET scans. J Nucl Med 2004;45: $1493-500$.

15. Roberts FO, Gunawardana DH, Pathmaraj K, Wallace A, $\mathrm{U}$ PL, Mi T, et al. Radiation dose to PET technologists and strategies to lower occupational exposure. J Nucl Med Technol 2005;33:44-7.

16. Zeff BW, Yester MV. Patient self-attenuation and technologist dose in positron emission tomography. Med Phys 2005; 32:861-5.

17. Brambilla M, Cannillo B, Dominietto M, Leva L, Secco C, Inglese E. Characterization of ordered-subsets expectation maximization with 3D post-reconstruction Gauss filtering and comparison with filtered backprojection in ${ }^{99 \mathrm{~m}} \mathrm{Tc}$ SPECT. Ann Nucl Med 2005;19:75-82. 